\title{
Main stem subocclusion shortly after aortic valve replacement
}

\author{
G Van Langenhove, P Van den Heuvel, F Van den Branden
}

A 58 year old woman was referred to our centre for aortic valve replacement because of a severe symptomatic aortic stenosis. She had no coronary risk factors. Rest ECG showed complete left bundle branch block. Coronary angiography showed normal coronary anatomy (fig 1). The gradient over the aortic valve could not be measured because of repetitive iatrogenic induction of ventricular tachycardia on inserting the catheter into the left ventricle. Non-invasive evaluation showed an echo Doppler gradient of $70 \mathrm{~mm} \mathrm{Hg}$ and an aortic valve area of $1.2 \mathrm{~cm}^{2}$ with normal left ventricular function. The patient was scheduled for aortic valve replacement.
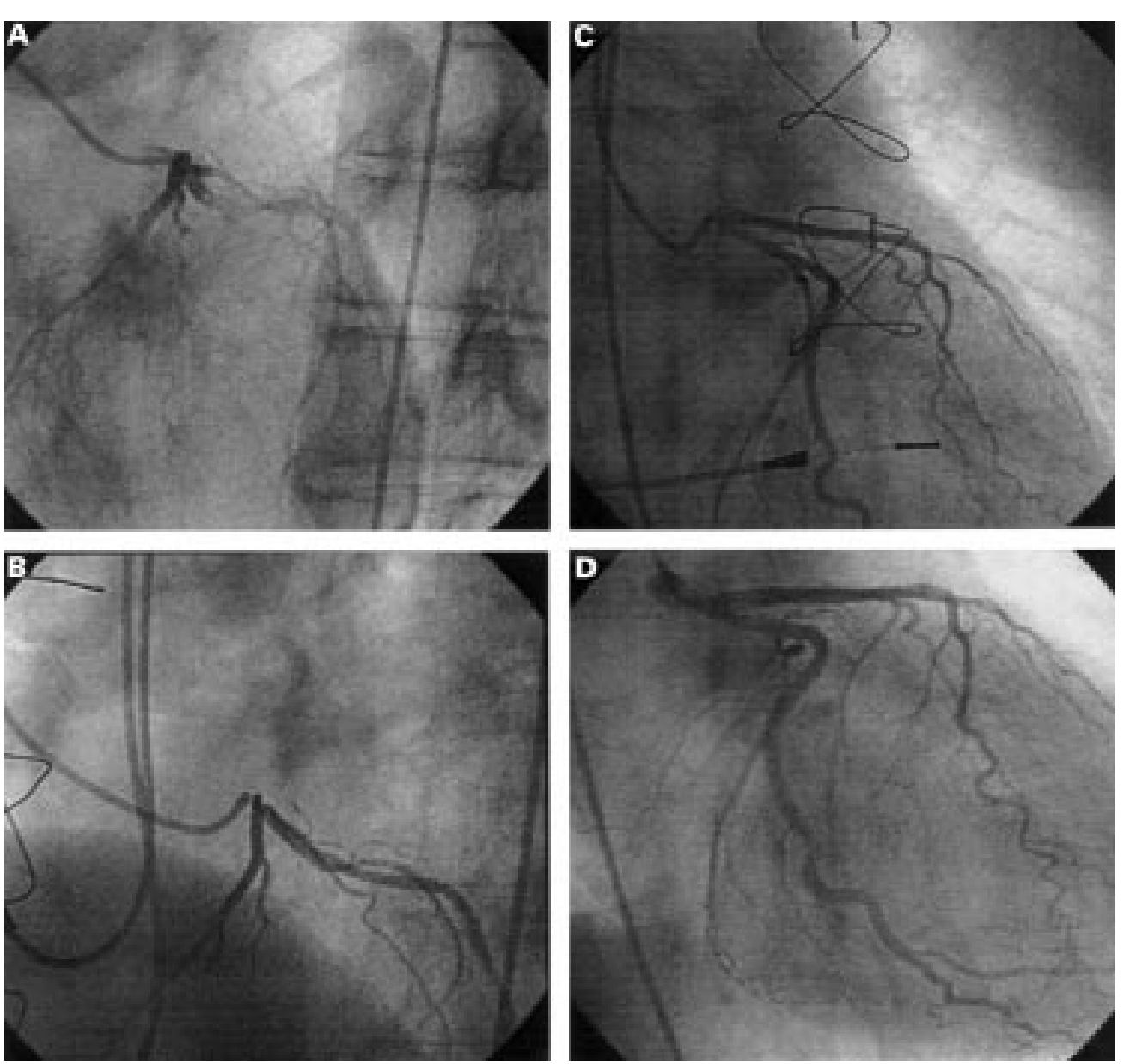

Figure 1 Coronary angiography showing (A) normal main stem (left anterior oblique view); (B) main stem stenosis (left anterior oblique view); (C) normal main stem (right anterior oblique view); (D) main stem stenosis (right anterior oblique view).

One litre of cold cardioplegia solution was given through antegrade cannulation of both coronary ostia. A St Jude Medical HP $23 \mathrm{~mm}$ valve was placed; the valve was attached with single stitches Ethibond 3.0 (Ethicon, Sommerville, New Jersey, USA). A few days after the procedure she developed atrial fibrillation with rapid ventricular response, which was successfully cardioverted. The rest of her stay was uneventful and she was discharged on an oral $\beta$ blocker, oral anticoagulation, and propafenone $150 \mathrm{mg}$ tid, which was stopped four weeks later.

She remained well until five months later, when on various occasions she presented with

Department of Cardiology,

Middelheim Hospital,

Lindendreef 1,2020

Antwerp, Belgium

$G$ Van Langenhove

$P$ Van den Heuvel

F Van den Branden

Correspondence to: Dr Van Langenhove.

Accepted for publication 22 April 1998 
exertional limitation, exhaustion, and atrial fibrillation, which each time was medically converted to sinus rhythm with intravenous propafenone. A submaximal cycloergometric scintigraphy with sestamibi showed minor ischaemia in the lateroseptal region; there also was a small fixed lesion in the apical region caused by attenuation. Echocardiographic Doppler examination showed normal function of the mechanical valve, again with normal left ventricular function.

Three weeks after the beginning of the symptoms, she was readmitted with signs of acute pulmonary oedema, without significant ECG changes. We decided on urgent coronary angiography, and found a $99 \%$ main stem stenosis with otherwise normal coronary vessels (fig 1). Urgent coronary bypass was performed. Six months later she is doing well, and has shown no signs of cardiac arrhythmia or chest discomfort.

Iatrogenic coronary artery stenosis is a known, albeit uncommon and often life threatening complication of aortic valve replacement. Main stem stenosis after aortic valve replacement was first recognised in $1970^{12}$; incidence may be as high as $0.9 \%{ }^{1}$ as reported by Lesage et al, however De Pace et al believe that incidence is sharply falling, because of better operative techniques. ${ }^{3}$

Symptoms are often clear and overwhelming: chest pain during exercise or at rest, sudden onset left ventricular failure without clear cause, and acute pulmonary oedema. Several pathogenic mechanisms have been suggested: aortic root fibrosis secondary to turbulent flow around the prosthesis; the presence of perfusion catheters during valve surgery that produce local pressure necrosis and subsequent intimal proliferation leading to obstruction of the coronary ostia; balloon inflation in the proximal parts of the vessels; turbulence that causes coronary artery intimal injury that might explain the lesions often found distant to the adherence of the cannulation devices. ${ }^{4}$

Bernhard et al showed that this complication can be avoided by not cannulating the coronary ostia for antegrade cardioplegia, but instead using retrograde delivery as an alternative method for myocardial perfusion during open heart surgery. ${ }^{5}$ In the same paper they suggested that there might be a genetic predisposition for developing such lesions, as $70 \%$ of the affected population in their series had an epsilon 4 allele apolipoprotein $\mathrm{E}$ genotype compared to $10-15 \%$ in a control group.

1 Lesage $\mathrm{CH}$, Vogel JH, Blount SG. Iatrogenic coronary occlusive disease in patients with prosthetic heart valves. Am $\mathcal{F}$ Cardiol 1970;26:123-9.

2 Roberts WC, Morrow AG. Late postoperative pathological findings after cardiac valve replacement. Circulation 1967; 35/36 (suppl 1):48-62.

3 De Pace NL, Lemole GM, Wolf NW, et al. Total left main coronary artery occlusion after aortic aneurysm repair and valve replacement. Chest 1991;99:515-17.

4 Sethi G, Scott S, Takaro T. Iatrogenic coronary artery stenosis following aortic valve replacement. f Thorac Cardiovasc Surg 1979;77:760-7.

5 Winkelmann BR, Ihnken K, Beyersdorf F, et al. Left main coronary artery stenosis after aortic valve replacement: genetic predisposition for accelerated arteriosclerosis after injury of the intact human coronary artery? Coron Artery Dis 1993;4:659-67. 